Pacific Journal of Mathematics

AN INTEGRAL REPRESENTATION FOR STRICTLY
CONTINUOUS LINEAR OPERATORS 


\section{AN INTEGRAL REPRESENTATION FOR STRICTLY CONTINUOUS LINEAR OPERATORS}

\section{W. BARTELT}

Let $B$ denote the algebra of bounded analytic functions on the open unit disc $D$ in the complex plane. Let $(B, \tau)$ denote $B$ endowed with the topology $\tau$, where $\tau$ is chosen from $\kappa, \beta$ or $\sigma$, respectively, the topology of uniform convergence on compact subsets of $D$, the strict topology and the topology of uniform convergence on $D$. This note obtains an integral representation of the form $T f(z)=\int_{\Gamma} f(w) K(z, w) d w$ where $\Gamma=\{z:|z|=1\}$ for the linear operators which are continuous from $(B, \kappa)$ into $(B, \sigma)$. This representation is then used to study the convergence of operators in the full algebra of all continuous linear operators from $(B, \beta)$ into $(B, \beta)$.

1. Introduction. Let $M(D)$ denote the set of bounded complex valued Borel measures on $D$. R. C. Buck [5] showed that $L$ is a continuous linear functional on $(C(D), \beta)$ if and only if $L f=\int_{D} f d \mu$, $\forall f \in C(D)$ for some $\mu \in M(D)$. L. A. Rubel and A. L. Shields [7] showed that for any $\mu \in M(D)$ there exists a function $h$ in $L^{\prime}(\Gamma)$ such that $\int_{D} f d \mu=\int_{\Gamma} f(x) h(x) d x, \forall f \in B$ and conversely, that any $h \in L^{1}(\Gamma)$ determines a measure $\mu \in M(D)$ for which this equality holds. Thus the continuous linear functionals on $(B, \beta)$ can be represented as integration over $\Gamma$ with respect to functions in $L^{\prime}(\Gamma)$.

Letting both $\tau_{1}$ and $\tau_{2}$ be one of the topologies $\kappa, \beta$ or $\sigma$, let $\left[\tau_{1}: \tau_{2}\right]$ denote the algebra of all continuous linear operators from $\left(B, \tau_{1}\right)$ into $\left(B, \tau_{2}\right)$.

In Theorem 1 it is shown that any linear operator $T$ in $[\beta: \beta]$ can be represented in the form

$$
T f(z)=\int_{\Gamma} f(w) K(z, w) d w, \quad \forall f \in B .
$$

However, a necessary and sufficient condition on $K(z, w)$ that such a $T$ be in $[\beta: \beta]$ is not known.

The algebra $[\kappa: \sigma]$ is a dense subalgebra of $[\beta: \beta]$ in the compact open topology. In Theorem 3 it is shown that a linear operator $T$ is in $[\kappa: \sigma]$ if and only if $T f(z)=\int_{\Gamma} f(w) K(z, w) d w$ where the kernel 
$K(z, w)$ satisfies certain fixed conditions. One can then associate with every linear operator in $[\beta: \beta]$ an explicit kernel $K(z, w)$. In $\S 4$, the convergence of linear operators in $[\beta: \beta]$ is characterized by using the convergence of the sequence of associated kernels. In the last section this convergence criterion is applied to the special type of operators in $[\beta: \beta]$ called multipliers.

2. Definitions. The topology $\sigma$, of uniform convergence on $D$, is defined by the norm

$$
\|f\|=\sup \{|f(z)|:|z|<1\}
$$

The topology $\kappa$, of uniform convergence on compact subsets of $D$, can be defined by the family of semi-norms

$$
\|f\|_{r}=\sup \{|f(z)|:|z|<r\}
$$

where $0<r<1$. The strict topology $\beta$ was introduced by R. C. Buck in [3] as a topology on the set of bounded continuous functions on a space. It is defined by the family of semi-norms

$$
|f|_{\phi}=\|f \phi\|, \phi \in C_{0}[D],
$$

the continuous functions on $D$ which vanish at infinity. The strict topology was first employed to study $B$ in [4]. For properties of $\beta$ and its relation to $\kappa$ and $\sigma$ see [3], [4], [5] and [7]. In particular, a sequence of functions $\left\{f_{n}\right\}$ in $B$ converges strictly to zero if and only if it is uniformly bounded and converges $\kappa$ (or pointwise) to zero. Also the $\beta$ bounded subsets of $B$ are precisely the $\sigma$ bounded subsets.

In [2] two appropriate topologies were employed to study $[\beta: \beta]$. From $[\sigma: \sigma]$, the subalgebra $[\beta: \beta]$ inherits the usual operator norm topology where

$$
\|T\|=\sup \{\|T f\|:\|f\| \leqq 1, f \in B\} .
$$

The second topology is that of uniform convergence on bounded subsets of $B$ which in fact is equivalent on $[\beta: \beta]$ to the compact open topology.

Definition. A net of operators $\left\{T_{\alpha}\right\}$ in $[\beta: \beta]$ converges uniformly on bounded subsets (witten u.b.) to $T$ if and only if given any $\beta$ open set $G$ in $B$ with $0 \in G$ and any $\beta$ bounded set $S$ in $B$, there exists an $\alpha^{\prime}$ such that if $\alpha>\alpha^{\prime}$, then $\left(T_{\alpha}-T\right)(S) \subseteq G$. 
For properties of $[\beta: \beta]$ in these two topologies see [2]. In particular the u.b. bounded subsets are precisely the norm bounded subsets and $[\beta: \beta]$ is a u.b. (and hence norm) closed subalgebra of $[\sigma: \sigma]$.

In should be observed [1] that the continuity classes $[\kappa: \sigma],[\beta: \sigma]$, $[\beta: \beta]$ and $[\sigma: \sigma]$ are in fact algebras, they are related by the proper inclusions $[\kappa: \sigma] \subset[\beta: \sigma] \subset[\beta: \beta] \subset[\sigma: \sigma]$, and $[\kappa: \sigma]$ is dense in $[\beta: \beta]$ in the u.b. topology, but in the norm topology $[\kappa: \sigma]$ is dense in only $[\beta: \sigma]$. In the study of the u.b. denseness of $[\kappa: \sigma]$ in $[\beta: \beta]$, the operators $T_{r}$ play a significant role. Given an operator $T$ in $[\beta: \beta]$, the operator $[T]_{r}$ (sometimes written $T_{r}$ ) is defined by $T_{r} f(z)=T\left(f_{r}\right)(z)$ where $f_{r}(z)=f(r z), f \in B$, and $0<r<1$. An operator $T_{r}$ is in $[\kappa: \sigma]$ and it is known [2] that $\left\{T_{r}\right\}$ converges u.b. to $T$ as $r \uparrow 1$.

Finally, a result of P. Hessler (see [1] or [6]) shows that a linear operator $T$ is in $[\beta: \tau]$ if and only if whenever a sequence $\left\{f_{n}\right\}$ in $B$ converges strictly to zero, it follows that $\left\{T f_{n}\right\}$ converges $\tau$ to zero, where $\tau$ is $\kappa, \beta$ or $\sigma$.

3. An integral representation. Let $z$ be a fixed point in $D$. Then given a linear operator $T$ in $[\beta: \beta]$, the linear functional $L$ defined on $B$ by $L f=T f(z)$ is a continuous linear functional on $(B, \beta)$. Therefore, $T f(z)=L f=\int_{\Gamma} f(w) K_{z}(w) d w$ for some function $K_{z}(w)$ in $L^{\prime}(\Gamma)$. It is difficult to determine the relationship between the various functions $K_{z}$ that is necessary and sufficient to ensure that $T f(z)=\int_{\Gamma} f(w) K(z, w) d w$ will represent an operator in $[\beta: \beta]$. The following gives a necessary condition and a different sufficient condition.

THEOREM 1. For any linear operator $T$ in $[\beta: \beta]$,

$$
T f(z)=\int_{\Gamma} f(w) K(z, w) d w, \quad \forall f \in B
$$

where $K\left(z^{\prime}, w\right)=K_{z^{\prime}}$ is in $L^{\prime}(\Gamma)$ for each $z^{\prime}$ in $D$ and the $L^{1}$ norms of all the functions $K_{z^{\prime}}$ are uniformly bounded.

If $K(z, w)$ satisfies the above necessary conditions and $K(z, w)$ is analytic in $D$ for each fixed $w$ in $\Gamma$ and bounded on $D \times \Gamma$, then any $T$ so defined is in $[\beta: \beta]$.

Proof. Let $T$ be in $[\beta: \beta]$. Then, as before, let $L_{z}(f)=T f(z)$ for $z$ fixed in $D$. Since $L_{z}(f)=\int_{\Gamma} f(w) K_{z}(w) d w$, we have $\left\|L_{z}\right\|=\left\|K_{z}\right\|_{L^{\prime}}$ 
and $\left|L_{z}(f)\right|=|T f(z)| \leqq\|T\|\|f\|$, where $\left\|K_{z}\right\|_{L^{\prime}}$ denotes the usual $L^{1}$ norm of $K_{z}$ on $\Gamma$. Hence $\left\|K_{z}\right\|_{L^{\prime}}=\left\|L_{z}\right\| \leqq\|T\|$ for each $z$ in $D$.

For the converse, define $T f(z)=\int_{\Gamma} f(w) K_{z}(w) d w=$ $\int_{\Gamma} f(w) K(z, w) d w$. Then $T f(z)$ is continuous in $D$ since

$$
\begin{gathered}
T f\left(z_{1}\right)-T f(z)=\int_{\Gamma} f(w)\left[K\left(z_{1}, w\right)-K(z, w)\right] d w \\
=\int_{\Gamma} f(w)\left(z_{1}-z\right)(2 \pi i)^{-1} \int_{\gamma} K(s, w)\left[\left(s-z_{1}\right)(s-z)\right]^{-1} d s d w
\end{gathered}
$$

where $\gamma$ is a circle in $D$ with center $z_{1}$ and containing $z$ in its interior and hence

$$
\left|T f\left(z_{1}\right)-T f(z)\right| \leqq\|f\|\left|z_{1}-z\right| \sup \left|\left(s-z_{1}\right)(s-z)\right|^{-1}\|K\|_{R}
$$

where $\|K\|_{R}$ is the sup of $|K(z, w)|$ taken over $R=D \times \Gamma$. Thus $\left|T f\left(z_{1}\right)-T f(z)\right|$ tends to zero as $z$ approaches $z_{1}$. Then for any triangle $\Delta \quad$ in $\quad D, \int_{\Delta} T f(z)=\int_{\Delta} \int_{\Gamma} f(w) K(z, w) d w=\int_{\Gamma} \int_{\Delta} f(w) K(z, w) d w=$ 0. By Morera's theorem, $\operatorname{Tf}(z)$ is analytic in $D$.

Now $\operatorname{Tf}(z)$ is a bounded function since

$$
|T f(z)| \leqq \int_{\Gamma}|f(w) K(z, w)| d|w| \leqq 2 \pi\left\|K_{z}\right\|_{L^{\prime}}\|f\| \leqq M\|f\|
$$

for all $z$ in $D$. If $\left\{f_{n}\right\}$ converges strictly to zero, then $T f_{n}(z)=L_{z}\left(f_{n}\right)$ converges to zero. Hence $\left\{T f_{n}\right\}$ converges pointwise to zero and is uniformly bounded, which implies $\left\{T f_{n}\right\}$ converges strictly to zero.

Note that additional conditions are imposed on $K(z, w)$ in the converse only to ensure that $\operatorname{Tf}(z)$ is analytic. Any $K(z, w)$ which satisfies the necessary conditions and makes $T f$ analytic will yield a $T$ in $[\beta: \beta]$. It is certainly not necessary that $K(z, w)$ be analytic in $z$ because for any function $h$ in $L^{1}(\Gamma), \quad T f(z)=\int_{\Gamma} f(w) h(w) d w=$ $\int_{\Gamma} f(w) K(z, w) d w$ is strictly continuous and $K(z, w)=h(w)$ need only be defined a.e..

We consider now the case when $C$ is some rectifiable curve inside $D$ and $T f(z)=\int_{C} f(w) K(z, w) d w$ with $K(z, w)$ in $L^{\prime}(C)$ for any $z$. As 
in the previous theorem, if $T$ is in $[\beta: \beta]$, then the functions $K_{z}(w)$ are uniformly bounded in $L^{\prime}(C)$ norm. Hence $T$ is in $[\kappa: \sigma]$, because if $\left\{f_{n}\right\}$ converges $\kappa$ to zero, then $\left\{f_{n}\right\}$ converges uniformly to zero on $C$ and $\left|T f_{n}(z)\right| \leqq$ (length of $C$ ) $\left\|f_{n}\right\|_{C}\left\|K_{z}\right\|_{L^{\prime}(C)}$.

Now we obtain a representation formula for the operators in $[\kappa: \sigma]$. Given an operator $T$ in $[\kappa: \sigma]$, there is an $M$ and an $r<1$ such that $\|T f\| \leqq M\|f\|_{r}$ for all $f$ in $B$. Letting $f(z)=z^{k}$, we obtain $\left\|T\left(z^{k}\right)\right\| \leqq M\left\|z^{k}\right\|_{r}=M(r)^{k}$. Hence $\left\|T\left(z^{k}\right)\right\|^{1 / k} \leqq r M^{1 / k}$ and lim sup $\left\|T\left(z^{k}\right)\right\|^{1 / k} \leqq r$.

THEOREM 2. If $T$ is in $[\kappa: \sigma]$, then there exists a function $K(z, w)$ analytic for $|z|<1$ and $\infty>|w|>r_{0}$ for some $r_{0}<1$ and such that if $1>r_{1}>r_{0}$, then there exists an $M$ such that $|K(z, w)| \leqq M$ for all $|z|<1$, $|w| \geqq r_{1}$ and such that

$$
T f(z)=\int_{|w|=r_{1}} f(w) \quad K(z, w) d w, \quad \forall f \in B .
$$

Conversely, using this representation formula, any such $K(z, w)$ yields an operator $T$ in $[\kappa: \sigma]$.

Proof. Explicitly the analyticity condition on the function $K(z, w)$ is that for $w$ fixed with $|w|>r_{0}, K(z, w)$ is an analytic function of $z$ for $z$ in $D$, and for $z$ fixed in $D, K(z, w)$ is an analytic function of $w$ in $\left\{w:|w|>r_{0}\right\}$.

Now let $K(z, w)$ satisfy the conditions of the theorem and put $T f(z)=(2 \pi i)^{-1} \int_{|w|=r_{1}} f(w) K(z, w) d w$. Then $T f(z)$ is analytic for $|z|<$ 1 just as in Theorem 1. Since $|T f(z)| \leqq M r_{1} \cdot\|f\|_{|w|=r_{1}}$, it follows that $T f$ is in $B$. Also $T$ is in $[\kappa: \sigma]$ since $\left\{f_{n}\right\}$ converging $\kappa$ to zero implies $\left\{f_{n}\right\}$ converges to zero uniformly on $|w|=r_{1}$.

Now assume that $T$ is in $[\kappa: \sigma]$ and let $K(z, w)=\sum_{k=0}^{\infty}\left(u_{k}(z) / w^{k+1}\right)$ where $T\left(z^{k}\right)=u_{k}$. For fixed $z$ in $D, \lim \sup \left|u_{k}(z)\right|^{1 / k} \leqq \lim \sup \left\|u_{k}\right\|^{1 / k}=$ $r_{0}$ for some real number $r_{0}<1$. Hence $\sup _{z \in D} \limsup \left|u_{k}(z)\right|^{1 / k} \leqq$ $r_{0}$. Hence $K(z, w)$ is analytic for $|w|>r_{0}$ for any fixed $z$ in $D$. Let $r_{1}$ be such that $1>r_{1}>r_{0}$. For large $k,\left\|u_{k}\right\| \leqq\left(r_{0}+\epsilon\right)^{k}$ with $r_{0}+\epsilon<r_{1}<1$ and hence for $|w|=r_{1},|z|<1,|K(z, w)| \leqq \sum_{k=0}^{\infty}\left(\left(r_{0}+\epsilon\right)^{k} /|w|^{k+1}\right)=$ $1 / r_{1} \sum_{k=0}^{\infty}\left(\left(r_{0}+\epsilon\right) / r_{1}\right)^{k}<\infty$. Now $K(z, w)$ is analytic in $D$ for fixed $\left|w_{0}\right|$ with $\left|w_{0}\right|>r_{0}$ because $\sum_{k=0}^{n}\left(u_{k}(z) / w_{0}^{k+1}\right)$ converges uniformly in $D$ to $K\left(z, w_{0}\right)$.

Now put $S f(z)=(2 \pi i)^{-1} \int_{|w|=r_{1}} f(w) K(z, w) d w$. Since $K(z, w)$ satisfies the conditions of the sufficiency part of the theorem, $S$ is in 
$[\kappa: \sigma]$. If $f(z)=z^{n}$, then

$$
S f(z)=(2 \pi i)^{-1} \int_{|w|=r_{1}} w^{n} \sum_{h=0}^{\infty}\left(u_{k}(z) / w^{h+1}\right) d w=u_{n}(z)=T\left(z^{n}\right) .
$$

Hence $S=T$ because they are both in $[\beta: \beta]$ and they agree on the polynomials, a $\beta$ dense subset of $B$.

Now that there is a representation for $T$ in $[\kappa: \sigma]$ on a curve inside the disk, the curve can be pushed to the boundary.

THEOREM 3. A linear operator $T$ is in $[\kappa: \sigma]$ if and only if

$$
T f(z)=\int_{\Gamma} f(w) K(z, w) d w, \quad \forall f \in B
$$

where $K(z, w)$ is analytic for $|w|>r_{0},|z|<1$ for some $r_{0}<1$ and if $1>r_{1}>r_{0}$, then there exists an $M$ such that $|K(z, w)| \leqq M$ for $|z|<1$ and $|w| \geqq r_{1}$.

Proof. Let $K(z, w)$ be given and put $S f(z)=(2 \pi i)^{-1}$ $\int_{|w|=r_{1}} f(w) K(z, w) d w$. Then by Theorem $2, S$ is in $[\kappa: \beta]$. Since $K(z, w)$ is analytic for $|w|>r_{0}$, it can be represented as $\sum_{k=0}^{\infty} g_{k}(z) / w^{k+1}$ where $g_{k}\left(z_{0}\right), k=0,1, \cdots$ is the sequence of coefficients in the series expansion of $K\left(z_{0}, w\right)$. Now $K(z, w)$ is bounded on $D \times \Gamma$ and analytic for $|z|<1$ for any fixed $w_{0}$ with $\left|w_{0}\right|=1$. Hence $K(z, w)$ satisfies the sufficiency conditions of Theorem 1. Let $T f(z)=$ $\int_{\Gamma} f(w) K(z, w) d w$. Then by Theorem $1, T$ is in $[\beta: \beta]$. But

$$
\begin{aligned}
S\left(z^{n}\right) & =\sum_{k=0}^{\infty} g_{k}(z)(2 \pi i)^{-1} \int_{|w|=r_{1}} w^{n} / w^{k+1} d w \\
& =g_{n}(z)
\end{aligned}
$$

and

$$
\begin{aligned}
T\left(z^{n}\right) & =(2 \pi i)^{-1} \int_{\Gamma} w^{n} \sum_{k=0}^{\infty}\left(g_{k}(z) / w^{k+1}\right) d w \\
& =\sum_{k=0}^{\infty} g_{k}(z)(2 \pi i)^{-1} \int_{\Gamma} w^{n} / w^{k+1} d w \\
& =g_{n}(z) .
\end{aligned}
$$


Since $S$ and $T$ agree on the polynomials and both are in $[\beta: \beta]$, they are equal. Hence $T$ is in $[\kappa: \sigma]$.

Let $T$ be in $[\kappa: \sigma]$ and put $K(z, w)=\sum_{k=0}^{\infty}\left(u_{k}(z) / w^{k+1}\right)$ where $T\left(z^{k}\right)=u_{k}(z)$. Then by Theorem $2, K(z, w)$ satisfies the conditions of Theorem 3 and hence of Theorem 1. Let $S f(z)=(2 \pi i)^{-1}$ $\int_{\Gamma} f(w) K(z, w) d w . \quad$ As above it follows that $S=T$.

Recall that if $T$ is an operator in $[\beta: \beta]$, then the operators $T_{r}$ for $0<r<1$ are in $[\kappa: \sigma]$ and $\left\{T_{r}\right\}$ converges uniformly on bounded subsets to $T$. This gives a limit representation for an operator in $[\beta: \beta]$. Are there any non-limiting representations of any operators in $[\beta: \beta]$ other than those in $[\kappa: \sigma]$ ?

Corollary. Let $T$ be in $[\beta: \beta]$. Then

$$
T f(z)=\lim _{r \uparrow 1} \int_{|w|=(1 / 2)(1+1 / r)} f_{r}(w) K(z, w) d w, \quad \forall f \in B
$$

where $K(z, w)=(2 \pi i)^{-1} \sum_{k=0}^{\infty}\left(T\left(z^{k}\right) / w^{k+1}\right) d w$.

Proof. Since $T_{r}$ is in $[\kappa: \sigma]$,

$$
\begin{aligned}
T_{r} f(z) & =(2 \pi i)^{-1} \int_{|w|=(1+r) / 2} f(w) \sum_{k=0}^{\infty}\left(T_{r}\left(z^{k}\right) / w^{k+1}\right) d w \\
& =(2 \pi i)^{-1} \int_{|w|=(1+r) / 2} f(w) \sum_{k=0}^{\infty}\left(T\left(z^{k}\right) r^{k} / w^{k+1}\right) d w \\
& =(2 \pi i)^{-1} \int_{|t|=(1+1 / r) / 2} f_{r}(t) \sum_{k=0}^{\infty}\left(T\left(z^{k}\right) / t^{k+1}\right) d t,
\end{aligned}
$$

by letting $w=r t$.

Now we use the integral representation for operators in $[\kappa: \sigma]$ to show that $[\kappa: \sigma]=\left\{T_{r}: T \in[\beta: \beta]\right\}$. This characterization of $[\kappa: \sigma]$ is a useful tool in the study of $[\kappa: \sigma]$ (see [2]).

TheOREM 4. $[\kappa: \sigma]=\left\{T_{r}: T \in[\beta: \beta], 0<r<1\right\}$.

Proof. We have to show that if $T$ is in $[\kappa: \sigma]$, then there exists an operator $S$ in $[\kappa: \sigma]$ and an $s<1$ such that $T=S_{s}$. Since $T$ is in $[\kappa: \sigma], \quad T f(z)=(2 \pi i)^{-1} \int_{|w|=r_{1}} f(w) K(z, w) d w$ where $K(z, w)=\Sigma_{k=0}^{\infty}$ $\left(T\left(z^{k}\right) / w^{k+1}\right)$ is analytic for $|w|>r_{0}$ and $1>r_{1}>r_{0}$. Let $K_{1}(z, w)=$ 
$s \sum_{k=0}^{\infty}\left(T\left(z^{h}\right) /(s w)^{h+1}\right)$ where $s<1$ and $r_{0}<r_{0} / s<1$. Define $S$ by $S f(z)=(2 \pi i)^{-1} \int_{|w|=s 1} f(w) K_{1}(z, w) d w$ where $1>s_{1}>r_{0} / s$. Then $S$ is in $[\kappa: \sigma]$ since $K_{1}(z, w)$ is analytic for $|z|<1,|w|>r_{0} / s$ and for $|z|<1$, $|w|=s_{1},\left|K_{1}(z, w)\right| \leqq s\left|\Sigma_{k=0}^{x}\left(T\left(z^{k}\right) /(s w)^{k+1}\right)\right|=s|K(z, s w)|<M$ since $r_{0}<s_{1} s=s|w|$. Let $\quad f(z)=z^{n}$. Then $\quad S f(z)=S f_{s}(z)=$ $(2 \pi i)^{-1} \int_{|w|=s_{1}}(s w)^{n} s \sum_{k=0}^{\infty}\left(T\left(z^{k}\right) /(s w)^{k+1}\right) d w=T(f)$ and hence $T=S_{s}$.

4. Convergence in $[\beta: \beta]$. In the Corollary to Theorem 3 of the last section it was shown that to any operator $T$ in $[\beta: \beta]$ there corresponds a kernel $K(z, w)$ by which $T$ is determined. Two operators $T_{1}$ and $T_{2}$ in $[\beta: \beta]$ should be close (e.g. $\left\|T_{1}-T_{2}\right\|$ small) if the corresponding kernels $K_{1}$ and $K_{2}$ are close (e.g. $\left\|K_{1}-K_{2}\right\|_{R}$ small for some region $R$ ).

However in relating $\left\|K_{1}-K_{2}\right\|_{R}$ to $\left\|T_{1}-T_{2}\right\|$ it seems that a suitable region $R$ can not be determined. For example if $T_{1}=0$ and $T_{2}=I$, the zero and identity operators respectively, then the kernel $K_{2}(z, w)$ corresponding to $I$ is $\sum_{k=0}^{\infty}\left(z^{k} / w^{k+1}\right)$ and

$$
\left\|K_{1}-K_{2}\right\|_{R}=\sup \left\{\left|\sum_{k=0}^{\infty}\left(z^{k} / w^{k+1}\right)\right|:|z|<1,|w|>1\right\}=\infty,
$$

where $R=\{(z, w):|z|<1,|w|>1\}$. On any region properly contained in $R$, uniform convergence of a sequence of functions $\left\{K_{n}\right\}$ is related to u.b. and not norm convergence of the corresponding operators $\left\{T_{n}\right\}$. One might be able to use $\left\|K_{1}-K_{2}\right\|_{R}$ where $R=$ $\{(z, w)=|z|<1,|w|>1\}$ if one considered only operators bounded away from $I$ in norm.

Obviously if $\left\{T_{n}\right\}$ and $T$ are in $[\beta: \beta]$ and the sequence of corresponding kernels $\left\{K_{n}\right\}$ converges to $K$ uniformly on $\{(z, w):|z|<$ $1,|w|>1\}$, then $\left\{T_{n}\right\}$ converges to $T$ in norm.

We will characterize the u.b. sequential convergence of operators in $[\beta: \beta]$ in terms of the corresponding kernels. Although the u.b. topology in $[\beta: \beta]$ is determined by the convergence of nets, the u.b. topology restricted to a norm (equivalently u.b.) bounded subset of $[\beta: \beta]$ is determined by sequential convergence [2].

The first step is to describe the u.b. convergence of a sequence of operators in $[\beta: \beta]$ in terms of their associated operators in $[\kappa: \sigma]$.

Let $C$ denote the algebra of functions in $B$ which are uniformly continuous on $D$. Recall that $\left[T_{n}\right]_{r} f=T_{n}\left(f_{r}\right)$ and observe that $T_{r}=T I_{r}$ where $I$ is the identity operator.

TheOrem 5. Let $\left\{T_{n}\right\}, n=1,2, \cdots$, and $T$ be linear operators in $[\beta: \beta]$. Then $\left\{T_{n}\right\}$ converges uniformly on bounded subsets to $T$ if and 
only if $\left[T_{n}\right]_{r}$ converges uniformly on bounded subsets to $T_{r}$ for every $0<r<1$ and there exists an $M$ such that $\left\|T_{n}\right\| \leqq M, n=1,2, \cdots$.

Proof. Let $\left\{T_{n}\right\}$ converge u.b. to $T$. Then $T_{n} f$ converges strictly to $T f$ for every fixed $f$ in $C$. Hence for fixed $f$ in $C,\left\{T_{n} f\right\}$ is uniformly bounded in norm, because strictly convergent sequences are bounded. By the uniform boundedness principle, the set $\left\{\left\|T_{n}\right\|\right\}$ is uniformly bounded, where $\left\|T_{n}\right\|=\sup \left\{\left\|T_{n} f\right\|: f \in C,\|f\| \leqq 1\right\}$. It follows [2] that this is the norm of $T_{n}$ as an operator on all of $B$.

Now fix $0<r<1$ and let $S$ be a bounded set and $G$ an open set in $(B, \beta)$. Then $\left(\left[T_{n}\right]_{r}-T_{r}\right)(S)=\left(T_{n}-T\right)\left(I_{r}\right)(S)=\left(T_{n}-T\right) S_{r} \subseteq G$ for $n>$ $N$ for some $N$, because $S_{r}=\left\{f_{r}: f \in S\right\}$ is a bounded set and $T_{n}$ converges u.b. to $T$.

For the converse let $G=\left\{g:|g|_{\psi}<3 \epsilon\right\}$ be an open set and $S$ a bounded set in $(B, \beta)$. Let $G_{1}=\left\{g:|g|_{\psi}<\epsilon\right\}$. For $f$ in $S$,

$$
\begin{aligned}
\left|\left(\left[T_{n}\right]_{r}-T_{n}\right) f\right|_{\psi} & =\left\|\psi\left(\left[T_{n}\right]_{r}-T_{n}\right) f\right\| \\
& =\left\|\psi T_{n}\left(I_{r}-I\right) f\right\| \\
& \leqq M\left\|\psi\left(I_{r}-I\right) f\right\| \\
& =M\left|\left(I_{r}-I\right) f\right|_{\psi} \\
& <\epsilon
\end{aligned}
$$

for $r \geqq r_{0}$ for some $r_{0}<1$ because $I_{r}$ converges u.b. to $I$. Hence for $r \geqq r_{0},\left(\left[T_{n}\right]_{r}-T_{n}\right) S \subseteq G_{1}$.

Since $T_{r}$ converges u.b. to $T$, there is an $r_{1}$ such that $1>r \geqq r_{1}$ implies $\left(T-T_{r}\right) S \subseteq G_{1}$. Fix $t$ larger than $r_{0}$ and $r_{1}$ and let $N$ be such that $n>N$ implies $\left(T_{t}-\left[T_{n}\right]_{t}\right) S \subseteq G_{1}$. Then for $n>N$,

$$
\begin{aligned}
\left(T-T_{n}\right) S & =\left(T-T_{t}\right) S+\left(T_{t}-\left[T_{n}\right]_{t}\right) S+\left(\left[T_{n}\right]_{t}-T_{n}\right) S \\
& \subseteq G_{1}+G_{1}+G_{1} \\
& \subseteq G
\end{aligned}
$$

Lemma 6. Let $\left\{T_{n}\right\}, n=1,2, \cdots$, and $T$ be in $[\beta: \beta]$. Then $\left[T_{n}\right]_{r}$ converges uniformly on bounded subsets to $T_{r}$ for every $0<r<1$ if and only if the corresponding kernels $K_{n}(z, w)$ converge to $K(z, w)$ uniformly on compact subsets of $D \times\{w:|w|>1\}$ and given $\rho>1$, there exists an $M_{\rho}$ such that $\left|K_{n}(z, w)\right| \leqq M_{\rho}$ for all $n$ and $|z|<1,|w| \geqq \rho>1$.

Proof. Let $\left[T_{n}\right]_{r}$ converge u.b. to $T_{r}$. Fix $r<1$ and $s<1$. Then it will be shown that $\left\{K_{n}(z, w)\right\}$ converges to $K(z, w)$ uniformly on $R=\{(z, w):|z| \leqq s$ and $|w| \geqq \rho>1 / r\}$. 
The operators $\left[T_{n}\right]_{r}$ and $T_{r}$ are maps from $C$ into $B$. As in the previous Theorem it follows that $\left\|\left[T_{n}\right]_{r}\right\|$ is uniformly bounded for $n=1,2, \cdots$.

Given $\epsilon>0$ and $s$ let $\psi$ in $C_{0}[D]$ be 1 on $\{z:|z|<s\}$. There is an $N$ such that for $n>N$ and for all $f$ in the bounded set $\{f:\|f\| \leqq 1\}$, $\left\|\left(\left[T_{n}\right]_{r}-T_{r}\right) f\right\|_{s} \leqq\left|\left(\left[T_{n}\right]_{r}-T_{r}\right) f\right|_{\psi}<\epsilon$ since $\left[T_{n}\right]_{r}$ converges u.b. to $T_{r}$. Thus for $\|f\| \leqq 1$,

$$
\begin{aligned}
\left\|\left(\left[T_{n}\right]_{r}-T_{r}\right) f\right\| & \leqq\left\|\psi\left(\left[T_{n}\right]_{r}-T_{r}\right) f\right\| \\
& =\left|\left(\left[T_{n}\right]_{r}-T_{r}\right) f\right|_{\psi} \\
& <\epsilon .
\end{aligned}
$$

For $j=0,1, \cdots$, let $f_{j}(w)=w^{j}, u_{j}=T\left(f_{j}\right)$ and $u_{j, n}=T_{n}\left(f_{j}\right)$. Then $\left[T_{n}\right]_{r} f_{j}(z)-T_{r} f_{j}(z)=r^{j} u_{j, n}(z)-r^{j} u_{j}(z)$. Hence $\left\|r^{j}\left[u_{j, n}(z)-u_{j}(z)\right]\right\|_{s}<\epsilon$ for $n>N$ for all $j=0,1, \cdots$. For $j=1,2, \cdots, J$, we have $\left\|u_{j . n}(z)-u_{j}(z)\right\|_{s}<\epsilon / r^{J}$ for $n>N$ since $r^{j} \geqq r^{J}$.

Now $K_{n}(z, w)-K(z, w)=\sum_{k=0}^{\infty}\left(u_{k, n}(z)-u_{k}(z)\right) r^{k} / w^{k+1} r^{k}$. For $n>$ $N,\left\|r^{k}\left[u_{k, n}(z)-u_{k}(z)\right]\right\|_{s}<\epsilon<1$. Since $r \rho>1$ there is a $J$ so large that $\sum_{k=J+1}^{\infty}(1 / r \rho)^{k}<\epsilon / 2$. Then

$$
\left\|K_{n}(z, w)-K(z, w)\right\|_{R} \leqq\left\|\sum_{k=0}^{J}\left(u_{k, n}(z)-u_{k}(z)\right) / w^{k+1}\right\|_{R}+\epsilon / 2
$$

since $1 /|w r| \leqq 1 / \rho r$. Also for $n>N,\left\|u_{k, n}(z)-u_{k}(z)\right\|_{s}<\epsilon / 2$ for $k=$ $1,2, \cdots, J$. Therefore $\left\|K_{n}-K\right\|_{R}<\epsilon$.

It remains to be shown that given $\rho>1$, there exists a constant $M_{\rho}$ such that $\left|K_{n}(z, w)\right| \leqq M_{\rho}$ for all $|z|<1$ and $|w|>\rho>1$. Given $\rho>1$, fix $0<r<1$ with $r \rho>1$. Since $\left[T_{n}\right]_{r}$ converges u.b. to $T_{r}$ it follows from Theorem 6 that there exists an $M$ with $\left\|\left[T_{n}\right]_{r}\right\| \leqq M$ for all $n=1,2, \cdots$. Now

$$
\begin{aligned}
K_{n}(z, w) & =\sum_{k=0}^{\infty} T_{n}\left(z^{k}\right) / w^{k+1} \\
& =r \sum_{k=0}^{\infty} r^{k} T_{n}\left(z^{k}\right) / r^{k+1} w^{k+1} \\
& =r \sum_{k=0}^{\infty}\left[T_{n}\right]_{r}\left(z^{k}\right) /(w r)^{k+1}
\end{aligned}
$$

and therefore for $|z|<1$ and $|w| \geqq \rho$,

$$
\begin{aligned}
\left|K_{n}(z, w)\right| & \leqq r M \sum_{k=0}^{\infty} 1 /|w r|^{k+1} \\
& \leqq M r \sum_{k=0}^{\infty}(1 /(\rho r))^{k+1}
\end{aligned}
$$

where the last expression is $\boldsymbol{M}_{\boldsymbol{\rho}}$. 
For the converse, fix $0<r<1$ and let $\gamma=(1+1 / r) / 2$. Now

$$
\begin{aligned}
\left\|\left(\left[T_{n}\right]_{r}-T_{r}\right) f\right\|_{s} & =\left\|\int_{|w|=\gamma} f_{r}(w)\left(K_{n}(z, w)-K(z, w)\right) d w\right\|_{s} \\
& \leqq\left\|K_{n}(z, w)-K(z, w)\right\|_{R}\|f\|
\end{aligned}
$$

where $R=\{(z, w):|z|<s, \quad|w|=(1+1 / r) / 2\}$. This last expression tends to zero as $n \rightarrow \infty$. Hence if $S$ is a bounded set, $\left(\left[T_{n}\right]_{r}-T_{r}\right) S$ converges $\kappa$ to zero.

Let $f$ in $B$ satisfy $\|f\| \leqq 1$. Then

$$
\begin{aligned}
\left\|\left[T_{n}\right]_{r} f(z)\right\| & =\left\|\int_{|w|=\gamma} f_{r}(w) K_{n}(z, w) d w\right\| \\
& \leqq\left\|K_{n}(z, w)\right\|_{R}\|f\| \\
& \leqq M
\end{aligned}
$$

by assumption on the kernels $K_{n}$ where $R=\{(z, w):|z|<1$, $|w|=(1+1 / r) / 2\}$. Hence $\left\|\left[T_{n}\right]_{r}\right\| \leqq M$ for all $n$.

Let $S$ be a bounded set in $(B, \beta)$ and let $G=\left\{g:|g|_{\psi}<\epsilon, \psi \neq \equiv 0\right\}$ be an open set in $(B, \beta)$. We have $\left\|\left(\left[T_{n}\right]_{r}-T_{r}\right)\right\| \leqq 2 M$. Let $r^{\prime}$ be such that for $|z|>r^{\prime},|\psi(z)|<\epsilon / 2 M$. Then

$$
\epsilon>\left\|\left(\left[T_{n}\right]_{r}-T_{r}\right) f \psi\right\|_{r^{\prime}<|z|<1}
$$

For $|z| \leqq r^{\prime}$, choose $N$ such that $n>N$ implies

$$
\left\|\left(\left[T_{n}\right]_{r}-T_{r}\right) f\right\|_{r^{\prime}}<\epsilon\|\psi\|^{-1} \text { for all } f \text { in } S \text {. }
$$

Then $\left\|\left(\left[T_{n}\right]_{r}-T_{r}\right) f \psi\right\|_{r^{\prime}}<\epsilon$ and $\left(\left[T_{n}\right]_{r}-T_{r}\right) f \in G$ for $n>N$ and all $f$ in $S$.

Theorem 5 and Lemma 6 taken together characterize u.b. convergence on bounded sets in $[\beta: \beta]$ in terms of the kernel functions $K(z, w)$.

TheOREM 7. Let $\left\{T_{n}\right\}, n=1,2, \cdots$ and $T$ be in $[\beta: \beta]$. Then $\left\{T_{n}\right\}$ converges u.b. to $T$ if and only if the corresponding kernels $\left\{K_{n}(z, w)\right\}$ converge uniformly on compact subsets of $D \times\{w:|w|>1\}$ to $K(z, w)$ and for any $\rho>1$, there exists a number $M_{\rho}$ such that $\left|K_{n}(z, w)\right| \leqq M_{\rho}$ for $|z|<1$ and $|w| \geqq \rho>1$.

Corollary. Let $S$ be a norm (equivalently u.b.) bounded subset of $[\beta: \beta]$. Let $\left\{T_{n}\right\}, n=1,2, \cdots$, and $T$ be in $S$ with corresponding 
kernels $\left\{K_{n}(z, w)\right\}$ and $K(z, w)$. Then $\left\{T_{n}\right\}$ converges u.b. to $T$ if and only if $\left\{K_{n}(z, w)\right\}$ converges uniformly on compact subsets of $D \times$ $\{w:|w|>1\}$ to $K(z, w)$.

Proof. The condition that $\left\{K_{n}(z, w)\right\}$ converges $\kappa$ to $K(z, w)$ on $D \times\{w:|w|>1\}$ is necessary by the above theorem. Let $T$ in $S$ imply $\|T\| \leqq M$. Then it follows that $\left|K_{n}(z, w)\right| \leqq M_{\rho}$ for $|z|<1$ and $|w|>\rho$ and the condition is also sufficient.

On the locally compact Hausdorff space $D \times\{w:|w|>1\}$, a sequence $\left\{K_{n}(z, w)\right\}$ converges strictly to a function $K(z, w)$, [5], if and only if $\left\{K_{n}(z, w)\right\}$ converges uniformly on compact subsets of $D \times$ $\{w:|w|>1\}$ to $K(z, w)$ and $\left|K_{n}(z, w)\right|$ is uniformly bounded on $D \times$ $\{w:|w|>1\}$. The next corollary follows immediately from the previous theorem, but it is not known if the converse holds. See Theorem 8 for a similar result.

Corollary. Let $\left\{T_{n}\right\}, n=1,2, \cdots$ and $T$ be in $[\beta: \beta]$ with corres ponding kernels $\left\{K_{n}(z, w)\right\}$ and $K(z, w)$. If $\left\{K_{n}(z, w)\right\}$ converges strictly to $K(z, w)$ on $D \times\{w:|w|>1\}$, then $\left\{T_{n}\right\}$ converges u.b. to $T$.

5. Convergence of multipliers. The characterization of u.b. convergence in the last section is applied to the multiplier operators.

Definition. A multiplier on $B$ is a linear operator $T$ such that there exists a sequence $\left\{c_{n}\right\}$ with the property that $T\left(\sum_{n=0}^{\infty} a_{n} z^{n}\right)=$ $\sum_{n=0}^{\infty} a_{n} c_{n} z^{n}$ for every function $\sum_{n=0}^{\infty} a_{n} z^{n}$ in $B$. It is known [1] that an operator $T$ is a multiplier from $B$ into $B$ if and only if the sequence $\left\{c_{n}\right\}$ is one side of the sequence of Fourier-Stieltjes coefficients of a bounded complex valued regular Borel measure $\mu$ on $\Gamma$ and also $\|\mu\|=$ $\|T\|$. Also if $T$ is a multiplier from $B$ into $B$, then $T$ is in $[\kappa: \kappa]$, a subalgebra of $[\beta: \beta]$. Let $\hat{\mu}(k)$ denote the $k$ th Fourier-Stieltjes coefficient of the measure $\mu$.

Clearly, if $\left\{T_{n}\right\}, n=1,2, \cdots$ and $T$ are multipliers in $[\beta: \beta]$, and $\left\{T_{n}\right\}$ converges in norm to $T$ then $\lim _{n \rightarrow \infty} \hat{\mu}_{n}(k)=\hat{\mu}(k)$ uniformly in $k$, where $\mu_{n}$ and $\mu$ are the measures associated with $T_{n}$ and $T$ respectively. In other words, the sequence of functions $\left\{\hat{\mu}_{n}\right\}$ defined on $P$, the nonnegative integers, converges uniformly to $\hat{\mu}$ on $P$. One expects then that for u.b. convergence the functions $\left\{\hat{\mu}_{n}\right\}$ will converge strictly to $\hat{\mu}$ on $P$. On the locally compact Hausdorff space $P$, a sequence of functions $\left\{\hat{\mu}_{n}\right\}$ converges strictly to a function $\hat{\mu}$ if and only if $\left\{\hat{\mu}_{n}\right\}$ is uniformly bounded and $\left\{\hat{\mu}_{n}\right\}$ converges uniformly on compact subsets to $\hat{\mu}$ [5], i.e., pointwise on $P$. 
Theorem 8. Let $\left\{T_{n}\right\}, n=0,1, \cdots$, and $T$ be multipliers from $B$ into $B$ with associated measures $\left\{\mu_{n}\right\}$ and $\mu$. Then $\left\{T_{n}\right\}$ converges $u . b$. to $T$ if and only if $\left\{\hat{\mu}_{n}\right\}$ converges strictly to $\hat{\mu}$.

Proof. For necessity we must show that there exists an $M$ such that $\left|\hat{\mu}_{n}(k)\right| \leqq M$ for all $n, k=0,1, \cdots$, and $\lim _{n \rightarrow \infty} \hat{\mu}_{n}(k)=\hat{\mu}(k), k=$ $0,1, \cdots$. Since $\left\{T_{n}\right\}$ converges u.b. to $T$ there is an $M$ such that $\left\|T_{n}\right\| \leqq M, n=0,1, \cdots$. Since $\left\|T_{n}\right\|=\left\|\mu_{n}\right\|,\left|\hat{\mu}_{n}(k)\right| \leqq\left\|\mu_{n}\right\| \leqq M$. Let $\hat{\mu}_{n}(k)=c_{n, k}$. Now since $\left\{T_{n}\left(z^{k}\right)\right\}$ converges strictly to $T\left(z^{k}\right)$, we have $\left\{c_{n, k} z^{k}\right\}$ converges strictly to $c_{k} z^{k}$ as $n \rightarrow \infty$. Hence $\lim _{n \rightarrow \infty} c_{n, k}=c_{k}$.

For the sufficiency part of the proof, let $|z| \leqq s<1$ and $|w| \geqq \rho>$ 1. Then

$$
\begin{aligned}
\left|K_{n}(z, w)-K(z, w)\right| & =\left|\sum_{k=0}^{\infty}\left(c_{n, k}-c_{k}\right) z^{k} / w^{k+1}\right| \\
& \leqq \sum_{k=0}^{\infty}\left|c_{n, k}-c_{k}\right|(s / \rho)^{k}
\end{aligned}
$$

Let $k^{\prime}$ be such that $\sum_{k=k^{\prime}}^{\infty}(s / \rho)^{k}<\epsilon / 4 M$ and let $N$ be so large that $n>N$ implies $\left|c_{n, k}-c_{k}\right|<\epsilon \rho / 2(s-\rho)$ for $k=0,1, \cdots, k^{\prime}$. Then for $|z|<s$ and $|w| \geqq \rho, \quad\left|K_{n}(z, w)-K(z, w)\right|<\epsilon$. Also $\left|\sum_{k=0}^{\infty}\left(c_{n, k} z^{k} / w^{k+1}\right)\right| \leqq$ $M \rho(\rho-1)^{-1}$ for all $|z|<1$ and $|w| \geqq \rho$.

The multipliers from $B$ into $B$ which are in the algebra $[\beta: \sigma]$ correspond to the absolutely continuous measures on $\Gamma[1]$. Let $\phi_{n}$ in $L^{\prime}(\Gamma)$ correspond to the multiplier $T_{n}$.

COROllary. Let $\left\{T_{n}\right\}, n=1,2, \cdots$ and $T$ be multipliers in $[\beta: \sigma]$. Then $\left\{T_{n}\right\}$ converges uniformly on bounded subsets to $T$ if and only if $\left\|\phi_{n}\right\|_{L^{\prime}} \leqq M$ and $\lim _{n \rightarrow \infty} \hat{\phi}_{n}(k)=\hat{\phi}(k)$.

Corollary. Let $\left\{T_{n}\right\}, n=1,2, \cdots$ and $T$ be multipliers in $[\beta: \sigma]$. If $\left\{\phi_{n}\right\}$ converges to $\phi$ in $L^{1}$, then $\left\{T_{n}\right\}$ converges u.b. to $T$.

\section{REFERENCES}

1. M. W. Bartelt, Multipliers and operator algebras on bounded analytic functions, Pacific J. Math., 37 (1971), 575-584.

2. - Approximation in operator algebras on bounded analytic functions, Trans. Amer. Math. Soc., 170 (1972), 71-83.

3. R. C. Buck, Operator algebras and dual spaces, Proc. Amer. Math. Soc., 3 (1952), 681-687.

4. Algebraic properties of classes of analytic functions, Seminars on Analytic Functions, Vol. II, Princeton (1957), 175-188.

5. - Bounded continuous functions on a locally compact space, Michigan Math. J., 5 (1958), 95-104. 
6. L. A. Rubel and J. V. Ryff, The bounded weak-star topology and bounded analytic functions, J. Functional Anal., 5 (1970), 167-183.

7. L. A. Rubel and A. L. Shields, The space of bounded analytic functions on a region, Ann. Ins. Fourier, (Grenoble) 16 (1966), 235-277.

Received December 5, 1973. This research was partially supported by NSF Grants GP-24182 and GU-2605.

Rensselaer Polytechnic Institute 



\section{Pacific Journal of Mathematics}

\section{Vol. 56, No. $1 \quad$ November, 1975}

Shimshon A. Amitsur, Central embeddings in semi-simple rings .......... 1

David Marion Arnold and Charles Estep Murley, Abelian groups, A, such

that $\operatorname{Hom}(A,---)$ preserves direct sums of copies of $A \ldots \ldots \ldots .$.

Martin Bartelt, An integral representation for strictly continuous linear

operators ................................... 21

Richard G. Burton, Fractional elements in multiplicative lattices......... 35

James Alan Cochran, Growth estimates for the singular values of

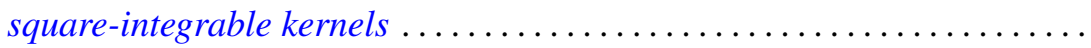

C. Martin Edwards and Peter John Stacey, On group algebras of central

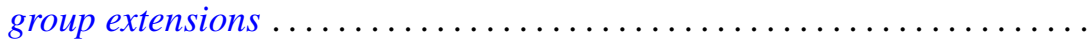

Peter Fletcher and Pei Liu, Topologies compatible with homeomorphism

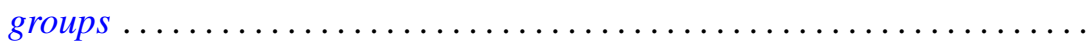

George Gasper, Jr., Products of terminating ${ }_{3} F_{2}(1)$ series ............ 87

Leon Gerber, The orthocentric simplex as an extreme simplex ............

Burrell Washington Helton, A product integral solution of a Riccati

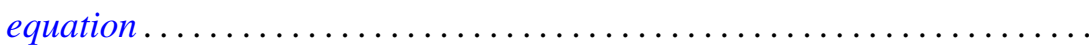

Melvyn W. Jeter, On the extremal elements of the convex cone of

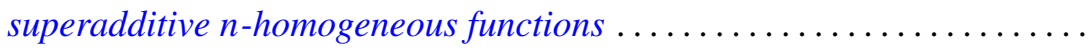

R. H. Johnson, Simple separable graphs .

Margaret Humm Kleinfeld, More on a generalization of commutative and

alternative rings. . .

A. Y. W. Lau, The boundary of a semilattice on an $n$-cell.

Robert F. Lax, The local rigidity of the moduli scheme for curves ...

Glenn Richard Luecke, A note on quasidiagonal and quasitriangular

operators .

Paul Milnes, On the extension of continuous and almost periodic functions

Hidegoro Nakano and Kazumi Nakano, Connector theory.

James Michael Osterburg, Completely outer Galois theory of perfect rings ..................................

Lavon Barry Page, Compact Hankel operators and the F. and M. Riesz

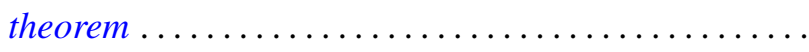

Joseph E. Quinn, Intermediate Riesz spaces..................... 225

Shlomo Vinner, Model-completeness in a first order language with a generalized quantifier.

Jorge Viola-Prioli, On absolutely torsion-free rings ..........

Philip William Walker, A note on differential equations with all solutions of integrable-square............................

Stephen Jeffrey Willson, Equivariant maps between representation 